\title{
Enhancement and Inhibition of Spontaneous Photon Emission by Resonant Silicon Nanoantennas
}

\author{
Dorian Bouchet, ${ }^{1}$ Mathieu Mivelle, ${ }^{1,2}$ Julien Proust, ${ }^{3}$ Bruno Gallas, ${ }^{4}$ Igor Ozerov, ${ }^{5}$ Maria F. Garcia-Parajo, ${ }^{2,6}$ \\ Angelo Gulinatti, ${ }^{7}$ Ivan Rech, ${ }^{7}$ Yannick De Wilde, ${ }^{1}$ Nicolas Bonod, ${ }^{3}$ Valentina Krachmalnicoff,,${ }^{1 *}$ and Sébastien Bidault ${ }^{1, \dagger}$ \\ ${ }^{1}$ ESPCI Paris, PSL Research University, CNRS, Institut Langevin, 1 rue Jussieu, F-75005 Paris, France \\ ${ }^{2}$ ICFO-Institut de Ciencies Fotoniques, The Barcelona Institute of Science and Technology, \\ 08860 Castelldefels (Barcelona), Spain \\ ${ }^{3}$ Aix-Marseille Univ, CNRS, Centrale Marseille, Institut Fresnel, 13013 Marseille, France \\ ${ }^{4}$ Sorbonne Universités, UPMC University Paris 06, CNRS UMR 7588, \\ Institut des Nanosciences de Paris, 75005 Paris, France \\ ${ }^{5}$ Aix-Marseille Univ, CNRS, CINAM, 13009 Marseille, France \\ ${ }^{6}$ ICREA-Institució Catalana de Recerca i Estudis Avançats, 08010 Barcelona, Spain \\ ${ }^{7}$ Politecnico di Milano, Dipartimento di Elettronica, Informazione e Bioingegneria, \\ Piazza da Vinci 32, 20133 Milano, Italy \\ (Received 11 May 2016; revised manuscript received 21 September 2016; published 28 December 2016)
}

Substituting noble metals for high-index dielectrics has recently been proposed as an alternative strategy in nanophotonics to design broadband optical resonators and circumvent the Ohmic losses of plasmonic materials. In this paper, we demonstrate that subwavelength silicon nanoantennas can manipulate the photon emission dynamics of fluorescent molecules. In practice, we show that dielectric nanoantennas can both increase and decrease the local density of optical states at room temperature, a process that is inaccessible with noble metals at the nanoscale. Using scanning probe microscopy, we analyze quantitatively, in three dimensions, the near-field interaction between a $100-\mathrm{nm}$ fluorescent nanosphere and silicon nanoantennas with diameters ranging between 170 and $250 \mathrm{~nm}$. Associated with numerical simulations, these measurements indicate increased or decreased total spontaneous decay rates by up to $15 \%$ and a gain in the collection efficiency of emitted photons by up to $85 \%$. Our study demonstrates the potential of silicon-based nanoantennas for the low-loss manipulation of solid-state emitters at the nanoscale and at room temperature.

DOI: 10.1103/PhysRevApplied.6.064016

\section{INTRODUCTION}

Broadband subwavelength optical resonators, or nanoantennas, have the ability to enhance the luminescence decay rate and brightness of solid-state emitters at room temperature by increasing the local density of optical states (LDOS) [1-8]. Generally based on noble metal nanoparticles exhibiting surface-plasmon polaritons, these resonators feature Ohmic losses that locally quench spontaneous photon emission and reduce fluorescence lifetimes $[2,3,8]$. Importantly, these nonradiative processes prevent plasmonic antennas from inhibiting spontaneous emission in their vicinity, as the LDOS can never be lower than the one found in vacuum. Even when plasmonic nanoantennas feature a dark mode weakly coupled to the far field, the nonradiative decay channels dominate and increase the decay rate of a coupled emitter $[9,10]$. In contrast, high-quality-factor dielectric cavities can exhibit a reduced LDOS to lengthen the excited-state lifetime of

\footnotetext{
valentina.krachmalnicoff@espci.fr

† sebastien.bidault@espci.fr
}

narrowband emitters such as isolated atoms [11] or solidstate light sources at cryogenic temperatures [12-15].

High-index dielectric nanoparticles featuring broadband low-order Mie resonances have been recently proposed as alternatives for the design of optical nanoantennas with weak Ohmic losses and strong magnetic modes [16-24], as well as enhanced local fields [25-29]. In particular, several theoretical studies have proposed using subwavelengthsized silicon resonators to accelerate spontaneous decay processes from isolated emitters [30-33]. In this work, we experimentally demonstrate that silicon nanoantennas can be used to resonantly enhance but also to inhibit spontaneous photon emission in fluorescent molecules at room temperature because of their low nonradiative losses. Furthermore, we show that these resonators increase the measured fluorescence signal by enhancing the collection efficiency of emitted photons through a transparent substrate, in excellent agreement with numerical simulations. In practice, the interaction between a silicon nanoantenna and solid-state emitters is fully characterized by tuning the position of a fluorescent nanosphere with nanometer precision in the vicinity of an $e$-beam-fabricated silicon disk using scanning probe microscopy. The continuous 


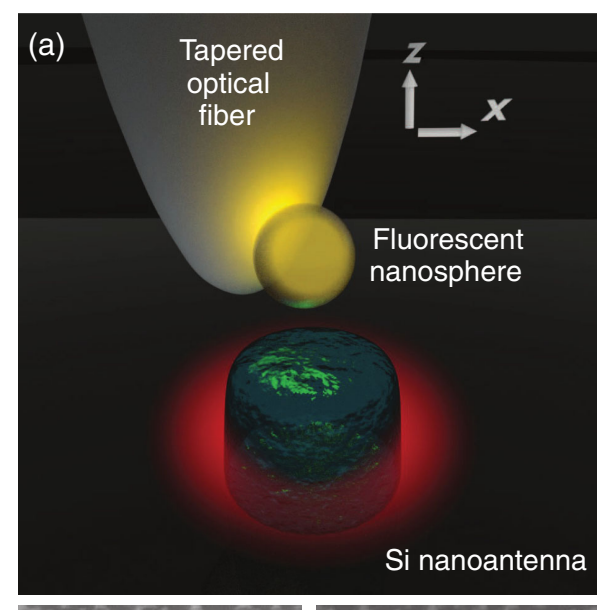

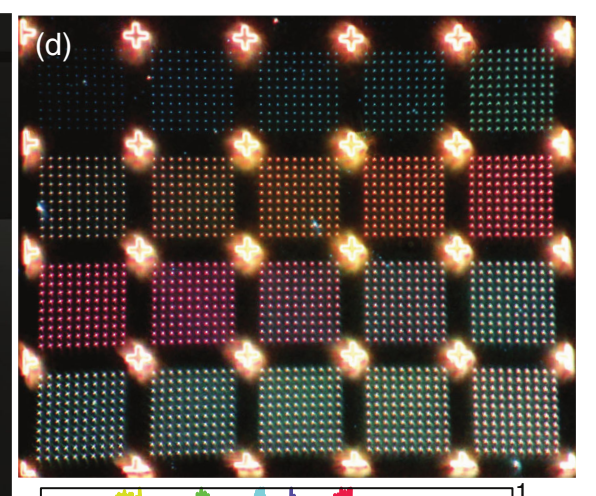

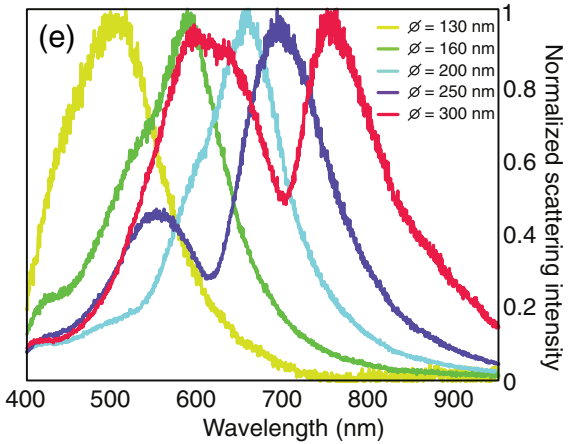

FIG. 1. (a) Sketch of the experimental study. SEM images of Si nanoantennas with base diameters of $170 \mathrm{~nm}$ (b) and $250 \mathrm{~nm}$ (c) (105 nm thickness). (d) Darkfield image of 20 arrays of $100 \mathrm{Si}$ disks with diameters ranging from $110 \mathrm{~nm}$ (upper left) to $300 \mathrm{~nm}$ (lower right). The distance between two consecutive disks in one array is $4 \mu \mathrm{m}$. (e) Typical scattering spectra of individual Si nanoantennas with diameters ranging from $130 \mathrm{~nm}$ to $300 \mathrm{~nm}$.
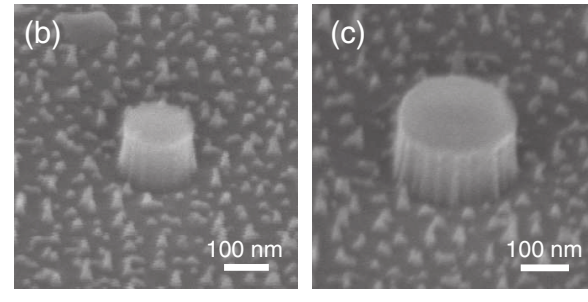

monitoring of the emission intensity and excited-state lifetime of the molecules then provides a three-dimensional map of the LDOS [34-36]. By experimentally showing how subwavelength-sized Si nanoantennas manipulate and engineer spontaneous light emission with low Ohmic losses, this study highlights the potential of dielectric resonators for nanoscale photon management.

\section{EXPERIMENTAL RESULTS AND DISCUSSION}

\section{A. Principle of the experiment}

To control the relative axial and lateral position of solid-state emitters with respect to a silicon nanoantenna, a 100-nm-diameter fluorescent sphere (Invitrogen Red FluoSpheres) is grafted at the extremity of a tapered optical fiber, which is mounted on a tuning fork to control its height using a sheer-force feedback loop. A Si nanoantenna is then scanned in three dimensions using a piezoelectric stage [Fig. 1(a)]. Nanometer-scale silicon disks are fabricated by electron-beam lithography and reactive ion etching in a 105-nm-thick amorphous Si film deposited on a 1-mm-thick silica substrate (see Appendix A). Figures 1(b) and 1(c) show scanning-electron-microscopy (SEM) images of two nanoantennas featuring base diameters of $170 \pm 5$ and $250 \pm 5 \mathrm{~nm}$, respectively. The etching process introduces a typical $10^{\circ}-15^{\circ}$ clearance angle between the base and the top of the fabricated silicon disks. In practice, the sample consists of 20 arrays of $100 \mathrm{Si}$ nanoantennas with diameters ranging from 110 to $300 \mathrm{~nm}$ (in increments of $10 \mathrm{~nm}$ ).

Choosing a film thickness close to $100 \mathrm{~nm}$ and tuning the nanoantenna diameters between 100 and $300 \mathrm{~nm}$ allows us to redshift the resonance wavelength of the first-order Mie modes (induced electric and magnetic dipoles) over the entire visible to near-infrared range [37-42]. This spectral evolution is evidenced in Figs. 1(d) and 1(e), which present a dark-field optical microscopy image of the entire sample and scattering spectra of selected Si nanoantennas, respectively. When varying the diameter from $110 \mathrm{~nm}$ (upper left) to $200 \mathrm{~nm}$ (second row right), the scattered light increases in intensity and shifts from the blue to the red part of the spectrum. As shown in Fig. 1(e), larger resonators (lowest two rows) feature quadrupolar resonances in addition to their dipolar modes, leading to broader scattering spectra and larger cross sections.

The absorption and emission spectra of the fluorescent nanosphere are centered on 580 and $610 \mathrm{~nm}$, respectively [see Fig. 2(a)]. In order to analyze Si nanoantennas in and out of resonance with the emitters, we consider two disk diameters of 170 and $250 \mathrm{~nm}$. As shown in Fig. 2(a), the smaller nanoantenna features a maximum scattering cross section corresponding to induced electric and magnetic dipoles [43] that overlap with the maximum of the fluorescence spectrum of the nanosphere. On the other hand, the dipolar and quadrupolar resonances of the 250-nm-diameter Si nanoantenna are centered on 550 and $710 \mathrm{~nm}$, respectively, with a local minimum of the scattering cross section around $610 \mathrm{~nm}$. These spectral properties are in very good agreement with the theoretical scattering cross sections shown in Fig. 2(b) obtained using finite-difference time-domain simulations (FDTDs) with the disk dimensions measured with SEM, the dielectric constant of the amorphous Si film estimated in ellipsometry, and considering a 10-nm-thick silica layer on the surface of the resonator due to the etching process [44], as well as an infinite silica substrate. 


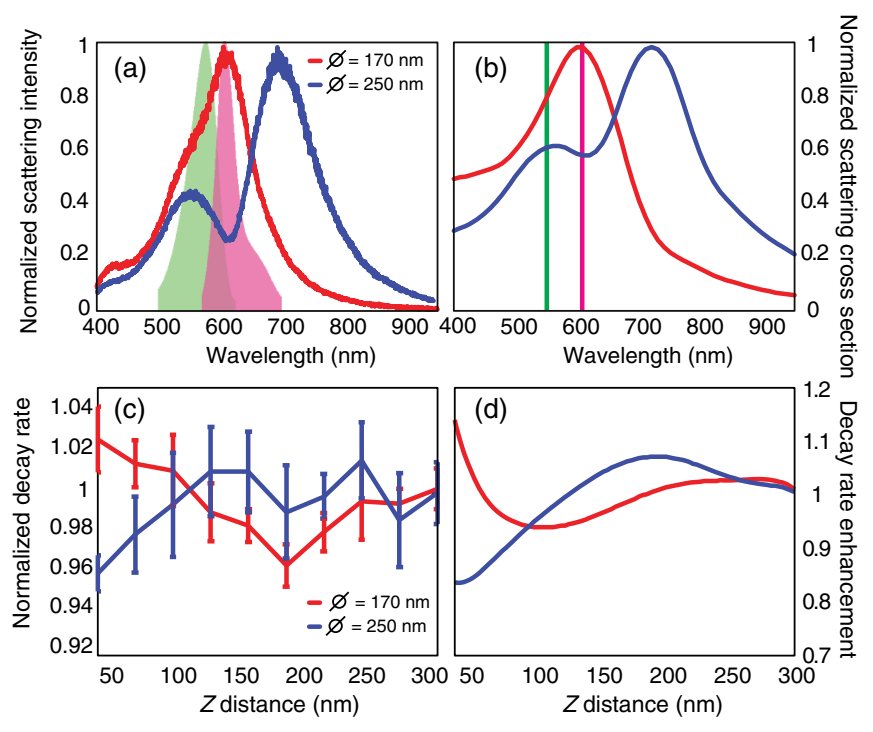

FIG. 2. Experimental (a) and simulated (b) scattering spectra of 170-nm-diameter (red solid line) and 250-nm-diameter (blue solid line) Si nanoantennas. The faint green and purple spectra superimposed in (a) correspond to the absorption and emission spectra of the fluorescent nanosphere. The green and purple vertical lines in (b) denote the excitation laser line and maximum of the fluorescence emission. Experimental (c) and theoretical (d) evolution of the emission decay rate as a function of the axial distance between the fluorescent nanosphere and the 170-nmdiameter (red data points and solid line) or 250-nm-diameter (blue data points and solid line) Si resonators.

\section{B. Spontaneous emission enhancement and inhibition}

Near-field optical measurements are performed in epifluorescence through the 1-mm silica substrate with a 0.7 -numerical-aperture (NA) objective used both for the 560-nm pulsed excitation (Fianium SC450, 40-ps pulses, $10-\mathrm{MHz}$ rate) and to collect the fluorescence signal at $610 \mathrm{~nm}$ on an avalanche photodiode (photocurrent detection of magnetic resonance, PDM-R, detector by Micro Photon Devices) [45] based on a recent silicon avalanche photodiode technology developed for enhanced sensitivity in the near infrared [46] and combined with a PicoQuant HydraHarp 400 acquisition board. The fluorescence lifetime and emission count rate of the nanosphere are measured while scanning the $\mathrm{Si}$ nanoantenna in three dimensions with respect to the tapered optical fiber. The lifetime $\tau$ is estimated for each position of the nanosphere using time-correlated single-photon counting and by fitting the decay curve with a single-exponential decay $[35,36]$. The measured decay rate $\Gamma=1 / \tau$, therefore, corresponds to an averaged value over the three orientations of the molecular transition dipoles and the spatial positions of approximately $10^{3}$ emitters in the 100-nm-diameter sphere [34].

Figure 2(c) shows the evolution of the excited-state decay rate of the fluorescent nanosphere when changing its axial position ( $z$ axis) with respect to the 170 - or $250-\mathrm{nm} \mathrm{Si}$ nanoantennas. The axial position corresponds to the distance between the top of the silicon disk and the bottom of the nanosphere when the latter is aligned with the center of the nanoantenna $(x=0)$. The change in $\Gamma$ is normalized with respect to the decay rate measured at $z=300 \mathrm{~nm}$. We monitor the oscillations of the decay rate similar to the ones observed when increasing the distance between a 2D interface and isolated solid-state emitters [47-50]. These damped oscillations are due to the constructive or destructive interference between the source dipoles in the nanosphere and the induced dipoles in the Si nanoantenna [48], whose phases depend on the emitter-resonator distance and the polarizability of the antenna [18,51]. Importantly, the oscillations in the vicinity of the "on-resonance" and the "off-resonance" antennas exhibit opposite behaviors: while the decay rate is increased close to the 170 -nm-diameter disk, it decreases in the vicinity of the 250-nm silicon nanoantenna. This measurement clearly highlights the interest of engineering the phase of the polarizability of dielectric resonators to influence spontaneous photon emission at the nanoscale. With plasmonic nanoantennas, the spectral dependence of the polarizability allows the control of emission directivity [51,52], but high Ohmic losses forbid the excited-state decay rate from being reduced in close proximity to the resonator, contrary to what is observed here with an off-resonance $250-\mathrm{nm} \mathrm{Si}$ nanoantenna. Lifetime oscillations similar to Fig. 2(c) can also be observed with planar films, but the interaction with fluorescent emitters is one dimensional, preventing the nanoscale engineering in three dimensions of inhibited or enhanced spontaneous photon emission.

To confirm the distance dependence of the emitterresonator interaction, we simulate in Fig. 2(d) the orientation-averaged decay-rate enhancement for an emitter in the center of the polystyrene sphere as a function of the axial distance $(z$ axis) to the resonator. FDTD simulations are performed for three orthogonal electric dipole sources emitting at $610 \mathrm{~nm}$, at the center of a 100-nm-diameter sphere with a refractive index of 1.57 and with the silicon disk and substrate properties used for the theoretical spectra of Fig. 2(b) (see Appendix B). In particular, the shear-force feedback control of the tapered fiber and the grafting process of the polystyrene sphere produces a slight offset of the minimum axial separation between the fluorescent nanosphere and the nanoantenna surface, which can be estimated from the simulations. The $z$ dependence of the lifetime oscillations simulated in Fig. 2(d) is in good agreement with the experimental data for both resonator diameters when considering a minimum of $35 \pm 5 \mathrm{~nm}$ for the axial separation between the polystyrene sphere and the resonator. Furthermore, compared to the experimental conditions, the FDTD simulations neglect spatial averaging over the dimensions of the polystyrene sphere. As further illustrated in the following paragraphs, the simulations considering a single-orientation-averaged emitter provide a good agreement with the experimentally measured spatial 
dependences of the fluorescence decay rate and intensity, although with higher amplitudes.

\section{Enhanced fluorescence collection efficiency}

The nanoscale dimensions of broadband silicon nanoantennas allow for a local modulation of fluorescence emission in three dimensions. To illustrate this, we plot in Fig. 3 the measured and simulated evolution of the fluorescence count rate when scanning the fluorescent nanosphere laterally over the 170 - or $250-\mathrm{nm}$ Si nanoantenna, with an estimated fixed axial separation of $120 \mathrm{~nm}$ with respect to the top of the resonator. We observe in Fig. 3(a) that the collected fluorescence intensity increases by $12 \%$ when the sphere is above the on-resonance silicon nanoantenna, compared to the count rate of the sphere without the antenna (at the same axial separation from the silica substrate). In contrast, the fluorescence intensity reduces by $40 \%$ on top of the 250 -nm-diameter disk. When increasing the lateral separation between the nanosphere and the Si nanoantenna, the collected intensity reduces by up to $38 \%$ and $60 \%$ for the smaller and larger disk, respectively, before recovering the intensity of the fluorescent sphere without the resonator at distances larger than $700 \mathrm{~nm}$.

In order to understand these results, we simulate twodimensional fluorescence intensity maps. The simulations require the computation of the excitation intensity at $560 \mathrm{~nm}$ as well as the emitter quantum yield and photon-collection efficiency at $610 \mathrm{~nm}$. The collection efficiency is estimated by taking into account the silica substrate and the limited NA of the microscope objective

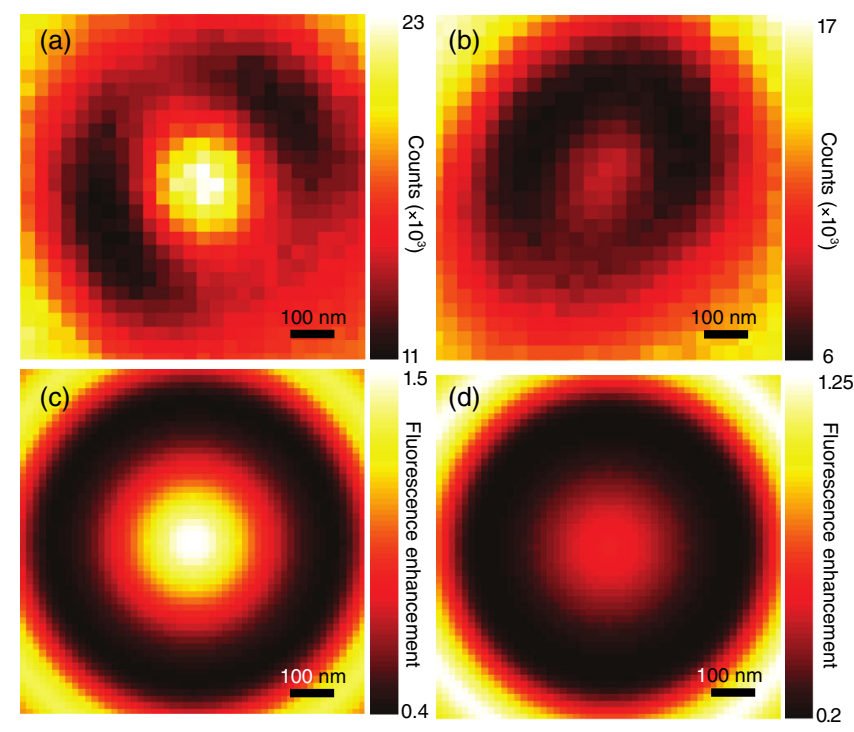

FIG. 3. Two-dimensional evolution of the measured (a),(b) and simulated (c),(d) collected fluorescence intensity in the vicinity of the 170-nm-diameter (a),(c) and 250-nm-diameter (b),(d) nanoantennas. The centers of the two-dimensional maps correspond to the nanosphere centered on the dielectric disks. while the initial quantum yield of the fluorescent emitter is set to 1 . The simulated fluorescence intensities in the sample plane given in Figs. 3(c) and 3(d) are in very good agreement with the experimental maps of Figs. 3(a) and 3(b). As mentioned above, the simulations do not consider the spatial distribution of fluorescent molecules in the sphere, which can account for the overestimated countrate increase on the 170-nm-diameter nanoantenna (the reduction with the off-resonance antenna is in good agreement with the experimental data). However, the numerical simulations allow the separate analysis of the excitation intensity, the luminescence quantum yield, and the photoncollection efficiency [Figs. 4(a)-4(c)]. As shown in Fig. 4(a), a 170-nm-diameter disk provides a $20 \%$ enhancement of the excitation intensity but not the off-resonance $\mathrm{Si}$ antenna. More importantly, the interaction between the emitter and a resonant Si nanoantenna leads to a $40 \%$ increase in the collection efficiency (from 10\% to 14\%) compared to the case of the fluorescent sphere without a resonator [Fig. 4(b)]. Inversely, an off-resonance antenna reduces the collected photon flux by $20 \%$. As shown in Fig. 4(d), the influence of the resonator size on the relative phase of the induced dipoles in the Si nanoantenna leads to changes in the angular radiation pattern in the silica substrate and, thus, to a reduced collection efficiency for the off-resonance case. On the other hand, the quantum yield of the emitter is similar in the vicinity of both antennas [Fig. 4(c)] with a $10 \%$ reduction because of the imaginary part of the dielectric constant of amorphous silicon. Finally, the reduced collected fluorescence intensity at distances of 200-300 $\mathrm{nm}$ from the resonators seen in Fig. 3 is related both to a reduction of the excitation intensity and to a lower percentage of photons emitted in the silica substrate.

\section{Nanoscale enhancement of spontaneous emission}

While Fig. 3 shows how silicon nanoantennas can modulate the collected fluorescence intensity, it does not demonstrate their ability to engineer the LDOS in three dimensions at the nanoscale. This is because with a fluorescent sphere located at $z=120 \mathrm{~nm}$, the lifetime of the emitters is similar for both resonator sizes [see Figs. 2(c) and 2(d)] and its fluctuations remain within the experimental error when scanning the $\mathrm{Si}$ nanoantenna laterally. We, thus, reduce the axial separation between the fluorescent nanosphere and the 170-nm-diameter $\mathrm{Si}$ antenna down to $35 \mathrm{~nm}$, which is the lowest value allowed by the shear-force feedback loop applied to the tapered optical fiber. At these short distances, a strong nanoscale modulation of the excited-state decay rate is observed [Figs. 5(a) and 5(b)]. In particular, both experimental and simulated values for the $2 \mathrm{D}$ spatial fluctuations of $\Gamma$ reveal an enhancement region centered on the nanoantenna with a typical size of $100 \mathrm{~nm}$ [Figs. 5(a) and 5(b)]. Furthermore, by radially averaging the spatial modulations of the 

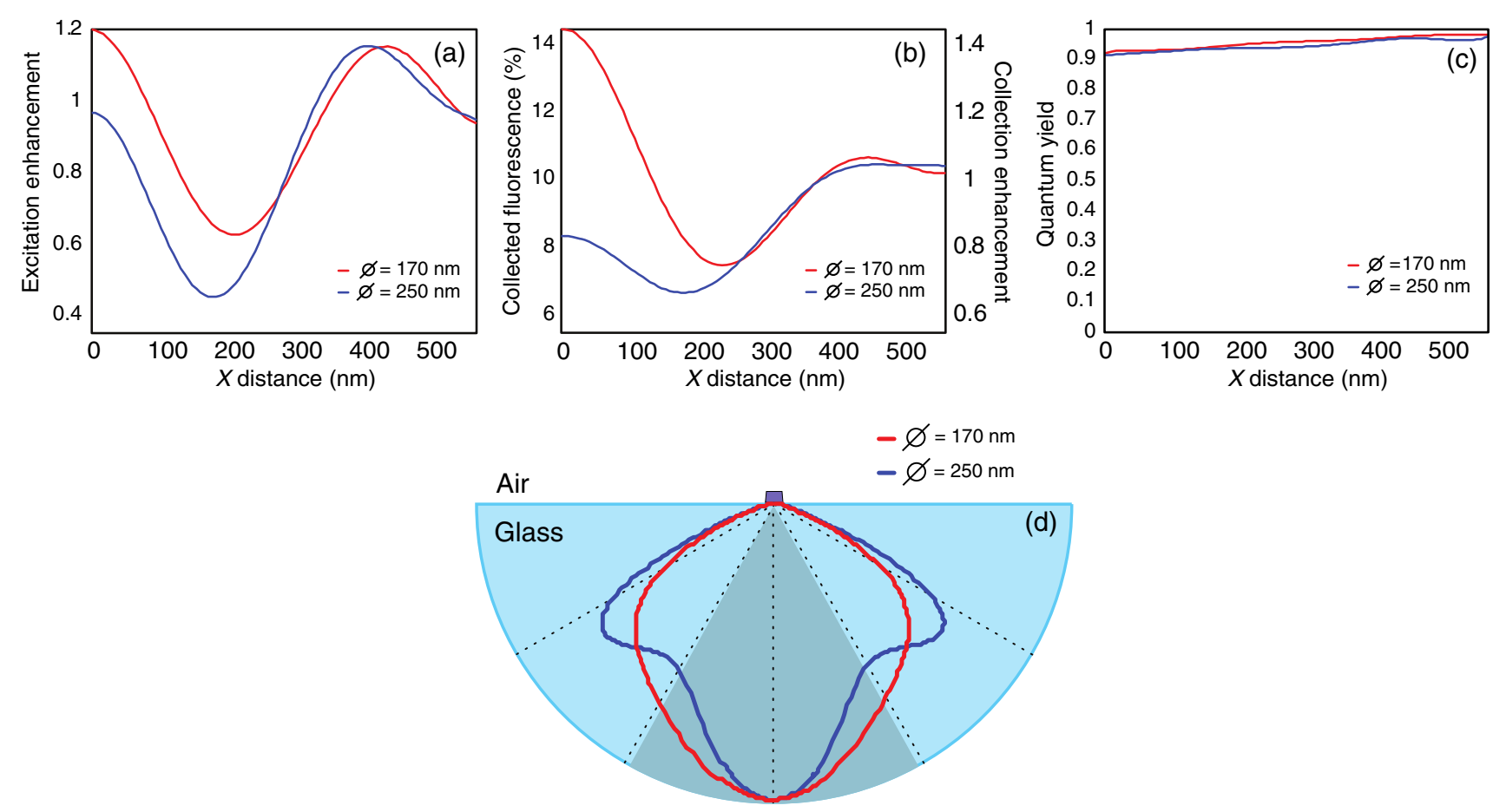

FIG. 4. Theoretical evolution of the excitation intensity enhancement in the center of the fluorescent sphere (a), of the fluorescence collection efficiency (b), and of the orientation-averaged quantum yield (c), as a function of the lateral distance between the nanosphere and the Si nanoantenna, with an axial separation of $120 \mathrm{~nm}$ above the 170-nm-diameter (red solid lines) or 250-nm-diameter (blue solid lines) resonator. $x=0$ corresponds to the nanosphere centered on the resonator. (d) Theoretical angular radiation pattern in the lower half-space when the fluorescent sphere is $120 \mathrm{~nm}$ above the 170-nm (purple solid line) or 250-nm (blue solid line) silicon nanoantenna. The darker part highlights the numerical aperture of the microscope objective.

fluorescence decay rate in Fig. 5(a), the line scan of Fig. 5(c) shows a $15 \%$ increase of $\Gamma$ at the center of the resonator $(x=0)$, which is in excellent agreement with the simulations provided in Fig. 5(d).

As a reference, Fig. 5(c) also shows the spatial evolution of the total decay rate when the axial separation between the sphere and the $170-\mathrm{nm}$ nanoantenna is $z=120 \mathrm{~nm}$. As already mentioned, the lifetime fluctuations are within the experimental error. Nevertheless, the fluorescence lifetime changes measured in Fig. 5(c) feature significantly weaker fluctuations as compared to the simulations of Fig. 5(d). This is again due to the spatial averaging of the LDOS changes over the fluorescent sphere diameter, which is neglected in the simulations. Importantly, both experiments and simulations for $z=35 \mathrm{~nm}$ [Figs. 5(c) and 5(d)] indicate a reduced LDOS at a lateral distance of $x \approx 120 \mathrm{~nm}$, demonstrating that the interference between the source dipoles and the dipoles induced in the $\mathrm{Si}$ nanoantenna lead to enhanced and inhibited spontaneous emission in the sample plane similar to what is observed along the $z$ axis in Fig. 2.

While changes in the axial separation between the sphere and the $\mathrm{Si}$ antenna strongly modify the total decay rate of the emitters, its influence on the measured fluorescence count rates is much weaker, as shown on Figs. 5(e) and 5(f). In particular, the solid red lines in Figs. 5(e) and 5(f) correspond to the radially averaged data of Figs. 3(a)-3(c). Both experimental data and simulations indicate similar damped spatial oscillations: the measured intensity is enhanced above the resonator but decreases at a lateral distance of about $200 \mathrm{~nm}$ before increasing again at larger spacings. As in Fig. 3, the simulated fluorescence count rate exhibits spatial fluctuations that are in good agreement with the experimental data, albeit with weaker amplitudes. Although lowering the position of the fluorescent sphere along the $z$ axis does not significantly influence the collected fluorescence signals shown in Figs. 5(e) and 5(f), numerical simulations indicate that it increases the photon-collection efficiency but reduces the excitation enhancement and the fluorescence quantum yield [Figs. 6(a)-6(c)]. In particular, reducing the axial distance of the fluorescent sphere above the resonator from 120 to $35 \mathrm{~nm}$ leads to a twofold increase in the collection efficiency enhancement [from $40 \%$ to $80 \%$; Fig. 6(b)] but decreases the excitation enhancement [from a $20 \%$ gain to a $10 \%$ reduction; Fig. $6(\mathrm{a})]$ and reduces the quantum yield [from 90\% to 80\%; Fig. 6(c)]. The enhanced collection efficiency and decreased quantum yield are expected when increasing the coupling between emitters and a resonator that radiates efficiently into the silica substrate but features some Ohmic losses. However, the reduced excitation intensity is more surprising. As shown 

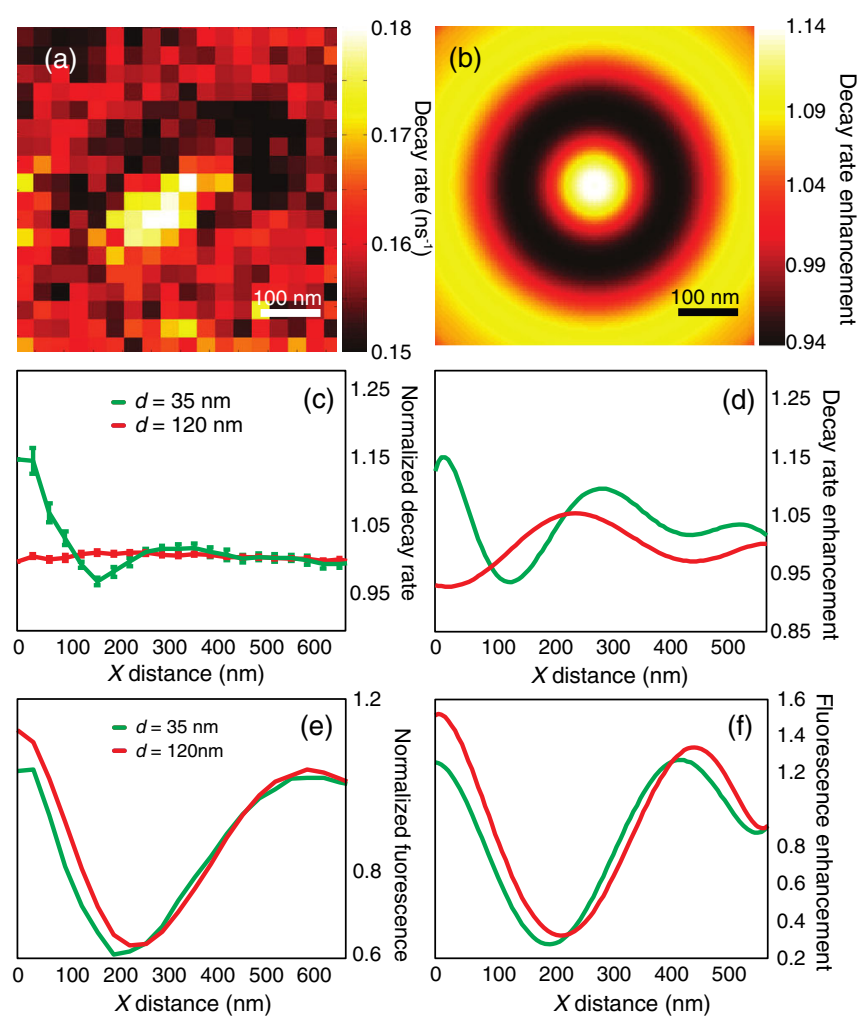

FIG. 5. Two-dimensional experimental (a) and simulated (b) maps of the total fluorescence decay rate in the sample plane, with an axial separation of $35 \mathrm{~nm}$. Evolution of the experimental (c),(e) and simulated (d),(f) fluorescence decay rate (c),(d) and intensity (e),(f) as a function of the lateral distance between the $170-\mathrm{nm}$ resonator and the fluorescent sphere for axial distances of $35 \mathrm{~nm}$ (green data points and solid lines) and $120 \mathrm{~nm}$ (red data points and solid lines). $x=0$ corresponds to the nanosphere centered on the resonator.

in Fig. 6(d), the local intensity is reduced for a fluorescent sphere centered above the 170-nm Si disk, and the excitation field can be only significantly enhanced on the sides of the resonator. While a lower excitation field intensity should lead to reduced radiative decay rates for fluorescent molecules in the sphere (because of reciprocity) [53], we measure enhanced total decay rates in Fig. 5 close to the on-resonance nanoantenna because the emitterresonator interaction is dominated by longitudinally oriented molecules [featuring a transition dipole perpendicular to the sample plane, see Fig. 6(e)], while the simulated local field enhancement of Fig. 6(d) is governed by transverse polarizations (in the sample plane).

\section{CONCLUSION}

Overall, the scanning probe technique used in this study allows us to characterize the resonant interaction between a fluorescent nanosphere and a silicon nanoantenna and to demonstrate inhibition and enhancement of spontaneous decay processes at the nanoscale and in three dimensions.
Furthermore, good agreement between electrodynamics simulations and experimental data proves that the resonant behavior of nanometer-scale silicon particles allows them to modulate the emission and far-field collection of fluorescence photons and makes them excellent candidates for the design of optical nanoantennas with low Ohmic losses. In particular, the simulated fluorescence intensity enhancements clearly show that the gain in the measured photon count rates for the resonant 170-nm-diameter Si nanoantenna is dominated by enhanced collection efficiency (by up to $85 \%$ ) and not by changes in excitation probability or by reciprocity of the local density of optical states (up to a $15 \%$ increase in the total spontaneous decay rate). This result highlights not only the interest of dielectric antennas to control the directivity of emitted photons but also the need to improve the near-field coupling between emitter and resonator. The larger field confinements expected in coupled silicon particles [26,29,30,32] associated with the top-down nanofabrication [52] or self-assembly strategies [6-8] developed for plasmonic nanoantennas will allow the optimized interaction between solid-state light sources and dielectricbased resonators.

\section{ACKNOWLEDGMENTS}

The authors thank F. Bedu for assistance on the sample fabrication. This work receives funding from the Laboratory of Excellence within the French program "Investments for the Future" under References No. ANR10-LABX-24 and No. ANR-10-IDEX-0001-02 PSL*, from Region Ile-de-France in the framework of DIM Nano-K, from the Programme Emergences 2015 of the City of Paris and from PSL Research University in the framework of project COSINE. Work at Institut Fresnel is carried out with the support of the A*MIDEX Project No. ANR-11-IDEX-0001-02 funded by the Investissements d'Avenir French Government program and managed by the French National Research Agency (ANR). M. M. and M. F. G.-P. gratefully acknowledge support from Fundacio Cellex and the Spanish Ministry of Economy and Competitiveness (Grant No. SEV-20150522). The nanofabrication processes are performed in the PLANETE cleanroom facility.

D. B. and M. M. contributed equally to this work.

\section{APPENDIX A: EXPERIMENTAL DETAILS}

\section{Sample fabrication}

A thin film of amorphous silicon is obtained by $e$-beam evaporation of a solid source on a 1-mm-thick UV-grade fused silica substrate in high vacuum. After chemical cleaning, the substrate is introduced in the growth chamber and undergoes a smooth plasma cleaning before deposition of the silicon layer at room temperature. The substrates are then transferred in a tubular furnace for a $1-\mathrm{h}$ annealing step at $600{ }^{\circ} \mathrm{C}$ in vacuum to increase the density of the silicon 

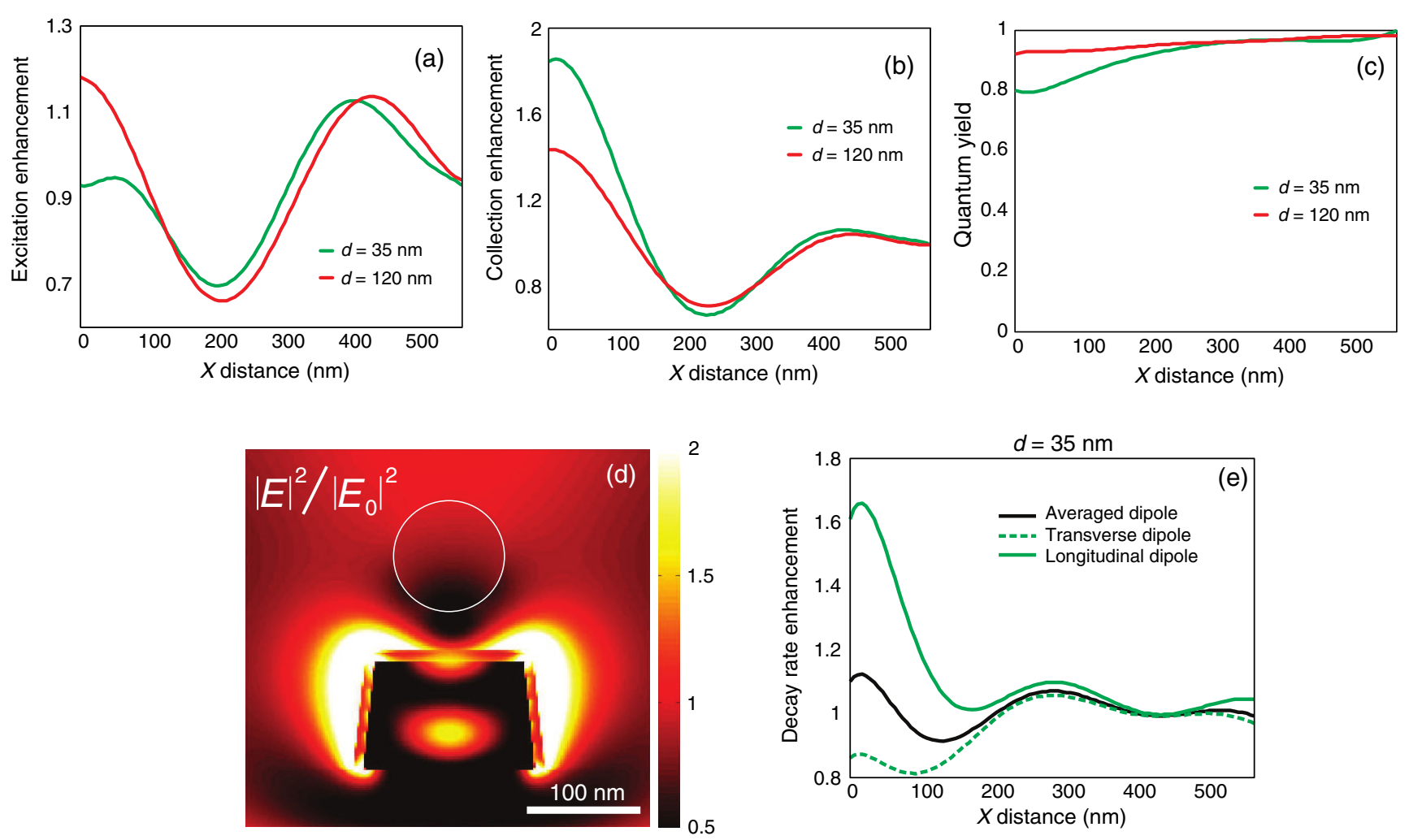

FIG. 6. Theoretical evolution of the excitation intensity enhancement in the center of the fluorescent sphere (a), of the fluorescence collection efficiency enhancement (b), and of the orientation-averaged quantum yield (c), as a function of the lateral distance between the nanosphere and the 170-nm-diameter Si nanoantenna, with an axial separation of $35 \mathrm{~nm}$ (green solid line) or $120 \mathrm{~nm}$ (red solid line). (d) Spatial distribution of the electric field intensity, in a longitudinal plane containing the 170-nm-diameter silicon antenna and the fluorescent sphere, for a 560-nm excitation wavelength (axial separation of $35 \mathrm{~nm}$ ). (e) Simulated decay rate enhancement for a longitudinal (solid green line), a transverse (dashed green line), or three orientationaveraged dipoles (solid black line) in the center of the sphere as a function of the lateral distance between the nanosphere and the 170-nm-diameter Si antenna, with an axial separation of $35 \mathrm{~nm}$.

thin film. The thickness $(105 \mathrm{~nm})$ and optical constants of the annealed films are determined ex situ by ellipsometry $(n=4.55+0.34 i$ at $610 \mathrm{~nm})$.

After oxygen plasma cleaning to remove organic residues and increase the wettability of the silicon surface, the annealed amorphous film is covered with a 60 -nm-thick layer of $e$-beam resist (PMMA diluted at $2 \%$ in ethyllactate, AR-P 679.04 from Allresist, Germany) by spin coating at $6000 \mathrm{rpm}$. After a soft baking process (10 min at $170{ }^{\circ} \mathrm{C}$ on a hot plate), a 30 -nm-thick conductive resist is spin coated on the $e$-beam resist (SX AR-PC 5000/90.1 from Allresist) and baked $2 \mathrm{~min}$ at $85^{\circ} \mathrm{C}$. The $e$-beam lithography is then performed by a field electron gun SEM system (PIONEER from RAITH). After exposure, the conductive resist is removed with deionized water, and the sample is dried under nitrogen. The development is performed for $60 \mathrm{~s}$ in a commercial solution [AR 600-55 from Allresist containing methyl isobutyl ketone and propan-2-ol (IPA)]. The development is stopped in IPA, and the sample dries under nitrogen. A 15-nm-thick nickel mask is then evaporated under vacuum (Auto 306 tool from Edwards). Nickel is used as the mask material because it is resistant to fluorine attacks [54]. A lift-off process in acetone using an ultrasonic bath removes the $e$-beam resist and the excess of nickel in areas of the sample that are not irradiated with the electron beam.

The nickel mask pattern is transferred into the amorphous thin silicon film by reactive ion etching (MG200, Plassys, France). The unprotected areas are etched by the plasma of a gas mixture containing $\mathrm{SF}_{6}, \mathrm{O}_{2}$, and $\mathrm{CHF}_{3}$ (respective fluxes of 20,8, and $5 \mathrm{sccm}$ ) alternated with a pure $\mathrm{O}_{2}$ plasma. Excited $\mathrm{SF}_{6}$ efficiently etches silicon, and the two key roles of the $\mathrm{CHF}_{3}$ gas are to passivate the vertical feature walls [54] and to etch the silicon oxide that forms during the process on the very reactive amorphous silicon surface. This configuration allows a very good etching anisotropy and nearly vertical walls for the fabricated structures. The rf power used to excite the plasma is $120 \mathrm{~W}$ for the gas mixture and $15 \mathrm{~W}$ for pure $\mathrm{O}_{2}$. This sequence is repeated as many times as required for the total etching of the thin silicon film. The nickel mask is removed by immersion in an aqueous $\mathrm{FeCl}_{3}$ solution ( $3 \mathrm{~min}$ ). Then the sample is rinsed by deionized water and dried under nitrogen flow. 


\section{Scanning electron microscopy and dark-field microscopy}

After all optical experiments are carried out, the sample featuring silicon resonators is covered by a 5-nm-thick titanium layer and imaged with the GEMINI scanning electron microscope (1-nm resolution) of a Zeiss Auriga 60 FIB-SEM. Dark-field images and spectra are measured in an inverted microscope (IX71, Olympus) coupled to a color CCD (Quicam, Roper) and to a fiber-coupled (50- $\mu \mathrm{m}$ core diameter) imaging spectrometer (Acton SP300 with PIXIS 100 CCD detector, Princeton Instruments). White light from a 100-W halogen lamp is focused on the sample using a (0.8-0.92)-NA dark-field condenser, and the scattered light is collected through the 1-mm-thick silica substrate with a $(60 \times 0.7)$-NA objective. The dark-field spectra of individual silicon nanoantennas are measured by aligning a single disk in the confocal volume corresponding to the $50-\mu \mathrm{m}$ fiber core of the imaging spectrometer with a 1-s acquisition time. A background spectrum is measured with the same acquisition time on an empty area of the sample. The background is subtracted to the measured spectra before correction according to the wavelength-dependent illumination and detection.

\section{Near-field optical measurements}

The sample is mounted on a sample-scanning inverted confocal microscope (Olympus, IX71). A fluorescent polystyrene sphere (Invitrogen Red FluoSpheres, $100 \mathrm{~nm}$ diameter) is grafted at the extremity of a tapered optical fiber (100-nm tip curvature) that is mounted on a tuning fork to control its height using a sheer-force feedback loop [35,36]. Pulsed laser excitation at $560 \mathrm{~nm}$ (Fianium SC450, 40-ps pulses, $10-\mathrm{MHz}$ rate) is focused on the sample through the silica substrate with a $0.7-\mathrm{NA} 60 \times$ objective. Fluorescence photons are collected through the same objective and are separated from the excitation with a dichroic mirror and a high-pass filter $(\lambda>580 \mathrm{~nm})$. Time-resolved photon detection is performed with a time-correlated single-photon counting system (PDMR detector by Micro Photon Devices combined with a PicoQuant HydraHarp 400 acquisition board), which allows one to simultaneously map the fluorescence intensity and decay rate (instrument response function with a $(190 \pm 30)$-ps full width at half maximum). The tapered optical fiber supporting the fluorescent sphere is held by shear-force feedback at a constant distance of approximately $20 \mathrm{~nm}$ to the surface of a nanostructured sample that is scanned in the $(x, y)$ plane (control electronics by RHK). The pixel size in the 2D intensity and decay rate maps is $30 \times 30 \mathrm{~nm}^{2}$ with a 1 -s acquisition time. The nanoscale drift of the experimental setup during the total measurement time of a $2 \mathrm{D}$ map (1000 s) explains the observed astigmatism in Figs. 3 and 5.

\section{Data analysis}

To estimate the data and error bars of Fig. 2(c), the evolution of the decay rate when changing the height of

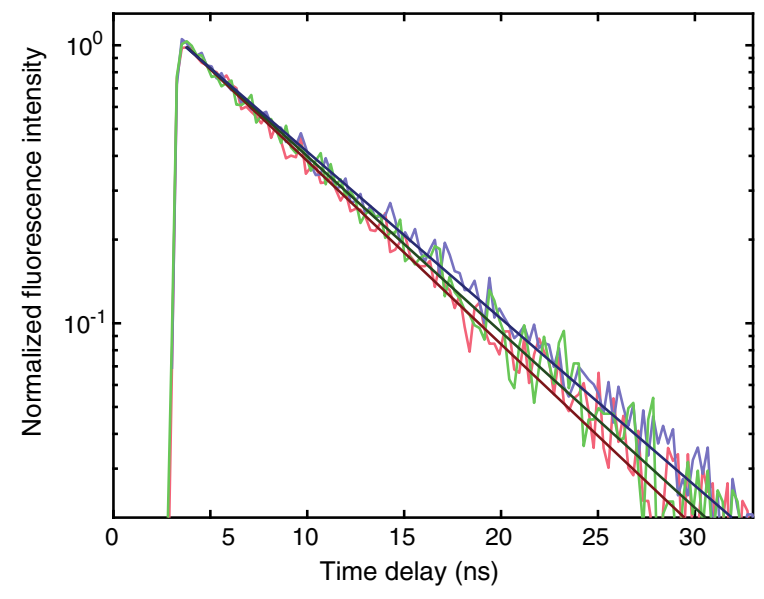

FIG. 7. Fluorescence intensity decay of a 100-nm nanosphere at an axial distance $z=35 \mathrm{~nm}$ from the $170-\mathrm{nm}$-diameter (red data points, $\tau=6.58 \pm 0.06 \mathrm{~ns}$ ) and 250-nm-diameter (blue data points, $\tau=7.25 \pm 0.05 \mathrm{~ns}$ ) $\mathrm{Si}$ nanoantennas. The green data points provide the intensity decay measured with an axial distance of $300 \mathrm{~nm}$ from the 170-nm antenna ( $\tau=6.85 \pm 0.08 \mathrm{~ns}$ ). The lifetimes are estimated from singleexponential fits (solid lines).

the fluorescent nanosphere is measured four (resp. three) consecutive times in the vicinity of the 170-nm-diameter (resp. $250 \mathrm{~nm}$ ) Si nanodisk. Each measurement consists in a series of ten successive acquisitions (10-s integration time) ranging from 35 to $300 \mathrm{~nm}$. Figure 7 shows typical fluorescence lifetime measurements close to the onresonance and off-resonance nanoantennas or with a large axial distance of $z=300 \mathrm{~nm}$. The experimental results presented in Fig. 2(c) are the estimated mean decay rates when fitting these data with a single-exponential decay, while the error bars correspond to the 95\% confidence interval (plus or minus twice the standard error). The mean decay rates are normalized with respect to the mean value estimated at $z=300 \mathrm{~nm}$.

The data of Figs. 5(c) and 5(e) are estimated by radially averaging the decay rate and intensity maps of Figs. 3(a) and 5(a). The center of the disk is first identified using the corresponding intensity map. All possible line scans going from the center of the disk to the edge of the map are then plotted, resulting in a set of measurements of the emission decay rate and intensity as a function of the distance from the resonator center in the sample plane. The experimental values presented in Fig. 5 are, for each distance, the mean decay rates and intensities, which are normalized with respect to the value estimated at $x=650 \mathrm{~nm}$. The error bars in Fig. 5(c) for each estimated decay rate correspond to the $95 \%$ confidence interval.

\section{APPENDIX B: NUMERICAL SIMULATIONS}

Numerical simulations are performed using an in-house FDTD code [55]. The model takes into account the 
nanoantenna dimensions estimated in SEM (105 nm thickness and 170 or $250 \mathrm{~nm}$ diameters), the dielectric constants of the amorphous silicon thin film measured by ellipsometry, and an infinite silica substrate. A 10-nm-thick layer of silicon dioxide $(n=1.5)$ is added around the silicon resonator to account for the oxidation of the semiconductor matrix after the RIE treatment. The fluorescent molecules in the polystyrene sphere are modeled by three dipoles oriented along the $x, y$, and $z$ axes, in the center of a 100-nm sphere with a dielectric index of 1.57. For simplicity, the tapered optical fiber is not taken into account. The simulations consider a volume spanning $\pm 2 \mu \mathrm{m}$ in $x, y$, and $z$ around the nanostructures. The antennas are surrounded by air (optical dielectric index of 1). All six boundaries of the computational volume are terminated with convolutional-periodic matching layers to avoid parasitic unphysical reflections around the nanostructures. The nonuniform grid resolution varies from $25 \mathrm{~nm}$, for areas at the periphery of the simulations, to $5 \mathrm{~nm}$ for the region in the immediate vicinity of the antennas.

Scattering spectra are simulated by impinging an unpolarized plane wave on the silicon nanoantenna from the upper air side of the sample. The scattered light is then collected in the silica lower half-space to account for the transmission configuration of the dark-field measurement.

Local excitation enhancement in the center of the sphere is estimated by impinging a plane wave propagating perpendicularly to the sample plane from the silica substrate on the sample. Changes in the total decay rate are obtained by computing the orientation-averaged changes in power dissipated by the three orthogonal dipoles in the center of the polystyrene sphere when changing its $x, y$, and $z$ position with respect to the silicon antenna. The orientation-averaged radiative-decay-rate evolution due to the resonator is estimated by projecting the Poynting vector produced by each of the three source dipoles on the surface of the computation volume. Finally, the collection efficiency is calculated by integrating the projected Poynting vectors on the limited numerical aperture of the microscope objective (solid angle with a $56^{\circ}$ width in the infinite silica substrate). For all calculations, we consider that the source electric dipoles have an intrinsic quantum yield of 1 and normalize the changes in local field distribution, as well as dissipated and radiated powers, with respect to a $100-\mathrm{nm}$ polystyrene sphere with a given $(z+105 \mathrm{~nm})$ height above the silica substrate, without the silicon antenna. Simulations are performed for each axial $(z$ axis) and lateral ( $x$ axis) position of the fluorescent nanosphere with respect to the center of the Si nanoantenna. The minimum considered axial distance $(35 \mathrm{~nm})$ is limited by the position of the fluorescent nanosphere on the tapered optical fiber and by the applied shear-force feedback loop during optical measurements. For shorter axial distances, strong variations of the excitation field and of the LDOS over the dimension of the nanosphere require simulating the emission properties of source dipoles at different positions of the nanosphere and not simply in its center.

[1] J. J. Greffet, Nanoantennas for light emission, Science 308, 1561 (2005).

[2] P. Anger, P. Bharadwaj, and L. Novotny, Enhancement and Quenching of Single-Molecule Fluorescence, Phys. Rev. Lett. 96, 113002 (2006).

[3] S. Kuhn, U. Hakanson, L. Rogobete, and V. Sandoghdar, Enhancement of Single-Molecule Fluorescence Using a Gold Nanoparticle as an Optical Nanoantenna, Phys. Rev. Lett. 97, 017402 (2006).

[4] M. Pfeiffer, K. Lindfors, C. Wolpert, P. Atkinson, M. Benyoucef, A. Rastelli, O. G. Schmidt, H. Giessen, and M. Lippitz, Enhancing the optical excitation efficiency of a single self-assembled quantum dot with a plasmonic nanoantenna, Nano Lett. 10, 4555 (2010).

[5] M. P. Busson, B. Rolly, B. Stout, N. Bonod, and S. Bidault, Accelerated single photon emission from dye moleculedriven nanoantennas assembled on DNA, Nat. Commun. 3, 962 (2012).

[6] G. P. Acuna, F. M. Moller, P. Holzmeister, S. Beater, B. Lalkens, and P. Tinnefeld, Fluorescence enhancement at docking sites of DNA-directed self-assembled nanoantennas, Science 338, 506 (2012).

[7] T. B. Hoang, G. M. Akselrod, C. Argyropoulos, J. N. Huang, D. R. Smith, and M. H. Mikkelsen, Ultrafast spontaneous emission source using plasmonic nanoantennas, Nat. Commun. 6, 7788 (2015).

[8] S. Bidault, A. Devilez, V. Maillard, L. Lermusiaux, J. M. Guigner, N. Bonod, and J. Wenger, Picosecond lifetimes with high quantum yields from single-photon-emitting colloidal nanostructures at room temperature, ACS Nano 10, 4806 (2016).

[9] M. Z. Liu, T. W. Lee, S. K. Gray, P. Guyot-Sionnest, and M. Pelton, Excitation of Dark Plasmons in Metal Nanoparticles by a Localized Emitter, Phys. Rev. Lett. 102, 107401 (2009).

[10] H. Mertens and A. Polman, Strong luminescence quantumefficiency enhancement near prolate metal nanoparticles: Dipolar versus higher-order modes, J. Appl. Phys. 105, 044302 (2009).

[11] R. G. Hulet, E. S. Hilfer, and D. Kleppner, Inhibited Spontaneous Emission by a Rydberg Atom, Phys. Rev. Lett. 55, 2137 (1985).

[12] E. Yablonovitch, Inhibited Spontaneous Emission in SolidState Physics and Electronics, Phys. Rev. Lett. 58, 2059 (1987).

[13] M. Bayer, T. L. Reinecke, F. Weidner, A. Larionov, A. McDonald, and A. Forchel, Inhibition and Enhancement of the Spontaneous Emission of Quantum Dots in Structured Microresonators, Phys. Rev. Lett. 86, 3168 (2001).

[14] S. Noda, M. Fujita, and T. Asano, Spontaneous-emission control by photonic crystals and nanocavities, Nat. Photonics 1, 449 (2007). 
[15] J. Bleuse, J. Claudon, M. Creasey, N. S. Malik, J. M. Gerard, I. Maksymov, J. P. Hugonin, and P. Lalanne, Inhibition, Enhancement, and Control of Spontaneous Emission in Photonic Nanowires, Phys. Rev. Lett. 106, 103601 (2011).

[16] A. B. Evlyukhin, C. Reinhardt, A. Seidel, B. S. Luk'yanchuk, and B. N. Chichkov, Optical response features of Si-nanoparticle arrays, Phys. Rev. B 82, 045404 (2010).

[17] A. Garcia-Etxarri, R. Gomez-Medina, L. S. Froufe-Perez, C. Lopez, L. Chantada, F. Scheffold, J. Aizpurua, M. NietoVesperinas, and J. J. Saenz, Strong magnetic response of submicron silicon particles in the infrared, Opt. Express 19, 4815 (2011).

[18] B. Rolly, B. Stout, and N. Bonod, Boosting the directivity of optical antennas with magnetic and electric dipolar resonant particles, Opt. Express 20, 20376 (2012).

[19] S. Person, M. Jain, Z. Lapin, J. J. Saenz, G. Wicks, and L. Novotny, Demonstration of zero optical backscattering from single nanoparticles, Nano Lett. 13, 1806 (2013).

[20] Y. H. Fu, A. I. Kuznetsov, A. E. Miroshnichenko, Y. F. Yu, and B. Luk'yanchuk, Directional visible light scattering by silicon nanoparticles, Nat. Commun. 4, 1527 (2013).

[21] I. Staude et al., Tailoring directional scattering through magnetic and electric resonances in subwavelength silicon nanodisks, ACS Nano 7, 7824 (2013).

[22] Y. M. Yang, W. Y. Wang, A. Boulesbaa, I. I. Kravchenko, D. P. Briggs, A. Puretzky, D. Geohegan, and J. Valentine, Nonlinear Fano-resonant dielectric metasurfaces, Nano Lett. 15, 7388 (2015).

[23] S. Makarov, S. Kudryashov, I. Mukhin, A. Mozharov, V. Milichko, A. Krasnok, and P. Belov, Tuning of magnetic optical response in a dielectric nanoparticle by ultrafast photoexcitation of dense electron-hole plasma, Nano Lett. 15, 6187 (2015).

[24] M. Decker and I. Staude, Resonant dielectric nanostructures: A low-loss platform for functional nanophotonics, J. Opt. 18, 103001 (2016).

[25] R. M. Bakker, D. Permyakov, Y. F. Yu, D. Markovich, R. Paniagua-Domínguez, L. Gonzaga, A. Samusev, Y. Kivshar, B. Luk'yanchuk, and A. I. Kuznetsov, Magnetic and electric hotspots with silicon nanodimers, Nano Lett. 15, 2137 (2015).

[26] J. van de Groep, T. Coenen, S. A. Mann, and A. Polman, Direct imaging of hybridized eigenmodes in coupled silicon nanoparticles, Optica 3, 93 (2016).

[27] H. Kallel, A. Arbouet, M. Carrada, G. Ben Assayag, A. Chehaidar, P. Periwal, T. Baron, P. Normand, and V. Paillard, Photoluminescence enhancement of silicon nanocrystals placed in the near field of a silicon nanowire, Phys. Rev. B 88, 081302 (2013).

[28] M. Caldarola, P. Albella, E. Cortes, M. Rahmani, T. Roschuk, G. Grinblat, R. F. Oulton, A. V. Bragas, and S. A. Maier, Non-plasmonic nanoantennas for surface enhanced spectroscopies with ultra-low heat conversion, Nat. Commun. 6, 7915 (2015).

[29] R. Regmi et al., All-dielectric silicon nanogap antennas to enhance the fluorescence of single molecules, Nano Lett. 16, 5143 (2016).

[30] B. Rolly, B. Bebey, S. Bidault, B. Stout, and N. Bonod, Promoting magnetic dipolar transition in trivalent lanthanide ions with lossless Mie resonances, Phys. Rev. B 85, 245432 (2012).

[31] M. K. Schmidt, R. Esteban, J. J. Saenz, I. Suarez-Lacalle, S. Mackowski, and J. Aizpurua, Dielectric antennas-A suitable platform for controlling magnetic dipolar emission, Opt. Express 20, 18609 (2012).

[32] P. Albella, M. A. Poyli, M. K. Schmidt, S. A. Maier, F. Moreno, J. J. Saenz, and J. Aizpurua, Low-loss electric and magnetic field-enhanced spectroscopy with subwavelength silicon dimers, J. Phys. Chem. C 117, 13573 (2013).

[33] X. Zambrana-Puyalto and N. Bonod, Purcell factor of spherical Mie resonators, Phys. Rev. B 91, 195422 (2015).

[34] M. Frimmer, Y. T. Chen, and A. F. Koenderink, Scanning Emitter Lifetime Imaging Microscopy for Spontaneous Emission Control, Phys. Rev. Lett. 107, 123602 (2011).

[35] V. Krachmalnicoff, D. Cao, A. Caze, E. Castanie, R. Pierrat, N. Bardou, S. Collin, R. Carminati, and Y. De Wilde, Towards a full characterization of a plasmonic nanostructure with a fluorescent near-field probe, Opt. Express 21, 11536 (2013).

[36] D. Cao, A. Cazé, M. Calabrese, R. Pierrat, N. Bardou, S. Collin, R. Carminati, V. Krachmalnicoff, and Y. De Wilde, Mapping the radiative and the apparent nonradiative local density of states in the near field of a metallic nanoantenna, ACS Photonics 2, 189 (2015).

[37] A. B. Evlyukhin, S. M. Novikov, U. Zywietz, R. L. Eriksen, C. Reinhardt, S. I. Bozhevolnyi, and B. N. Chichkov, Demonstration of magnetic dipole resonances of dielectric nanospheres in the visible region, Nano Lett. 12, 3749 (2012).

[38] A. I. Kuznetsov, A. E. Miroshnichenko, Y. H. Fu, J. B. Zhang, and B. Luk'yanchuk, Magnetic light, Sci. Rep. 2, 492 (2012).

[39] L. Shi, J. T. Harris, R. Fenollosa, I. Rodriguez, X. T. Lu, B. A. Korgel, and F. Meseguer, Monodisperse silicon nanocavities and photonic crystals with magnetic response in the optical region, Nat. Commun. 4, 1904 (2013).

[40] U. Zywietz, A. B. Evlyukhin, C. Reinhardt, and B. N. Chichkov, Laser printing of silicon nanoparticles with resonant optical electric and magnetic responses, Nat. Commun. 5, 3402 (2014).

[41] M. Abbarchi, M. Naffouti, B. Vial, A. Benkouider, L. Lermusiaux, L. Favre, A. Ronda, S. Bidault, I. Berbezier, and N. Bonod, Wafer scale formation of monocrystalline silicon-based Mie resonators via silicon-on-insulator dewetting, ACS Nano 8, 11181 (2014).

[42] J. Proust, F. Bedu, B. Gallas, I. Ozerov, and N. Bonod, Alldielectric colored metasurfaces with silicon Mie resonators, ACS Nano 10, 7761 (2016).

[43] J. van de Groep and A. Polman, Designing dielectric resonators on substrates: Combining magnetic and electric resonances, Opt. Express 21, 26285 (2013).

[44] J. Proust, F. Bedu, S. Chenot, I. Soumahoro, I. Ozerov, B. Gallas, R. Abdeddaim, and N. Bonod, Chemical alkaline etching of silicon Mie particles, Adv. Opt. Mater. 3, 1280 (2015).

[45] A. Giudice, G. Simmerle, D. Veronese, R. Biasi, A. Gulinatti, I. Rech, M. Ghioni, and P. Maccagnani, Highdetection efficiency and picosecond timing compact detector modules with red-enhanced SPADs, Proc. SPIE Int. Soc. Opt. Eng. 8375, 83750P (2012). 
[46] A. Gulinatti, I. Rech, F. Panzeri, C. Cammi, P. Maccagnani, M. Ghioni, and S. Cova, New silicon SPAD technology for enhanced red-sensitivity, high-resolution timing and system integration, J. Mod. Opt. 59, 1489 (2012).

[47] K. H. Drexhage, Interaction of light with monomolecular dye layers, Prog. Opt. 12, 163 (1974).

[48] R. R. Chance, A. Prock, and R. Silbey, Molecular fluorescence and energy transfer near interfaces, Adv. Chem. Phys. 37, 1 (1978).

[49] M. Frimmer and A. F. Koenderink, Spontaneous Emission Control in a Tunable Hybrid Photonic System, Phys. Rev. Lett. 110, 217405 (2013).

[50] M. Frimmer, A. Mohtashami, and A. F. Koenderink, Nanomechanical method to gauge emission quantum yield applied to nitrogen-vacancy centers in nanodiamond, Appl. Phys. Lett. 102, 121105 (2013).
[51] B. Rolly, B. Stout, S. Bidault, and N. Bonod, Crucial role of the emitter-particle distance on the directivity of optical antennas, Opt. Lett. 36, 3368 (2011).

[52] A. G. Curto, G. Volpe, T. H. Taminiau, M. P. Kreuzer, R. Quidant, and N.F. van Hulst, Unidirectional emission of a quantum dot coupled to a nanoantenna, Science 329, 930 (2010).

[53] L. Novotny and B. Hecht, Principles of Nano-Optics (Cambridge University Press, Cambridge, England, 2012).

[54] S. Franssila, Introduction to Microfabrication (J. Wiley, Chichester, England, 2004).

[55] M. Mivelle, T. Grosjean, G. W. Burr, U. C. Fischer, and M. F. Garcia-Parajo, Strong Modification of magnetic dipole emission through diabolo nanoantennas, ACS Photonics 2, 1071 (2015). 